Supporting Information

\title{
Tunable Band-Edge Potentials and Charge Storage in Colloidal Tin-Doped Indium Oxide (ITO) Nanocrystals
}

\author{
Jose J. Araujo, Carl K. Brozek, ${ }^{\dagger}$ Hongbin Liu, ${ }^{\dagger}$ Anna Merkulova, \\ Xiaosong Li, and Daniel R. Gamelin* \\ Department of Chemistry, University of Washington, Seattle, WA 98195-1700 \\ ${ }^{\dagger}$ Present address: Department of Chemistry and Biochemistry, University of Oregon, \\ Eugene, OR 97403 \\ †resent address: Microsoft Quantum, Redmond, WA 98052
}

Email:gamelin@uw.edu 
Nanocrystal Size Analysis
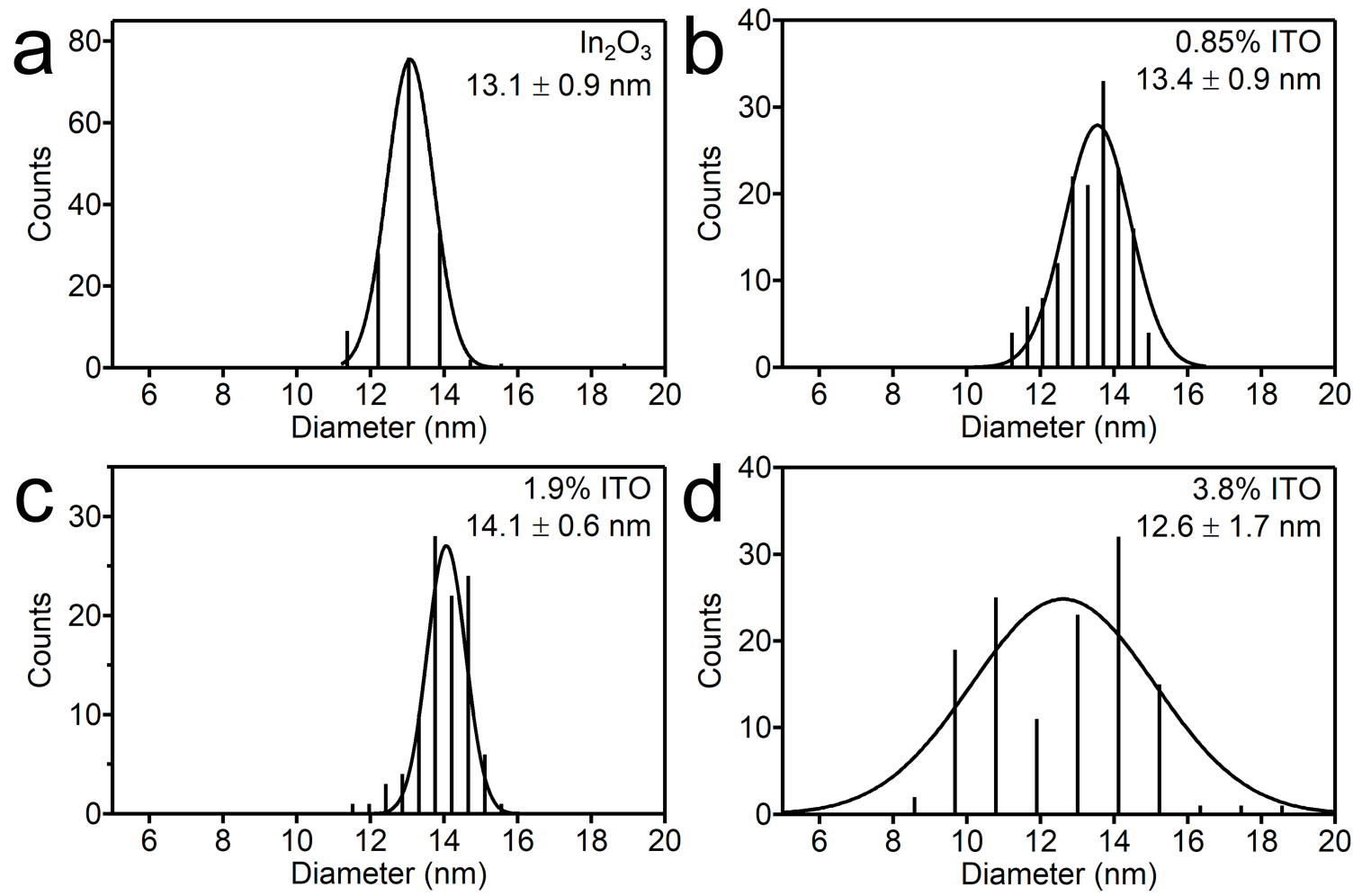

Figure S1. Histograms of NC diameters obtained from TEM analysis of (a) $\operatorname{In}_{2} \mathrm{O}_{3}$, (b) $0.85 \%$ ITO, (c) $1.9 \%$ ITO, and (d) $3.8 \%$ ITO NCs. Over 100 NCs were measured for each histogram. Each histogram was fitted with a Gaussian, and the average diameter and standard deviation are indicated in each plot. 


\section{Spectroelectrochemical Data}
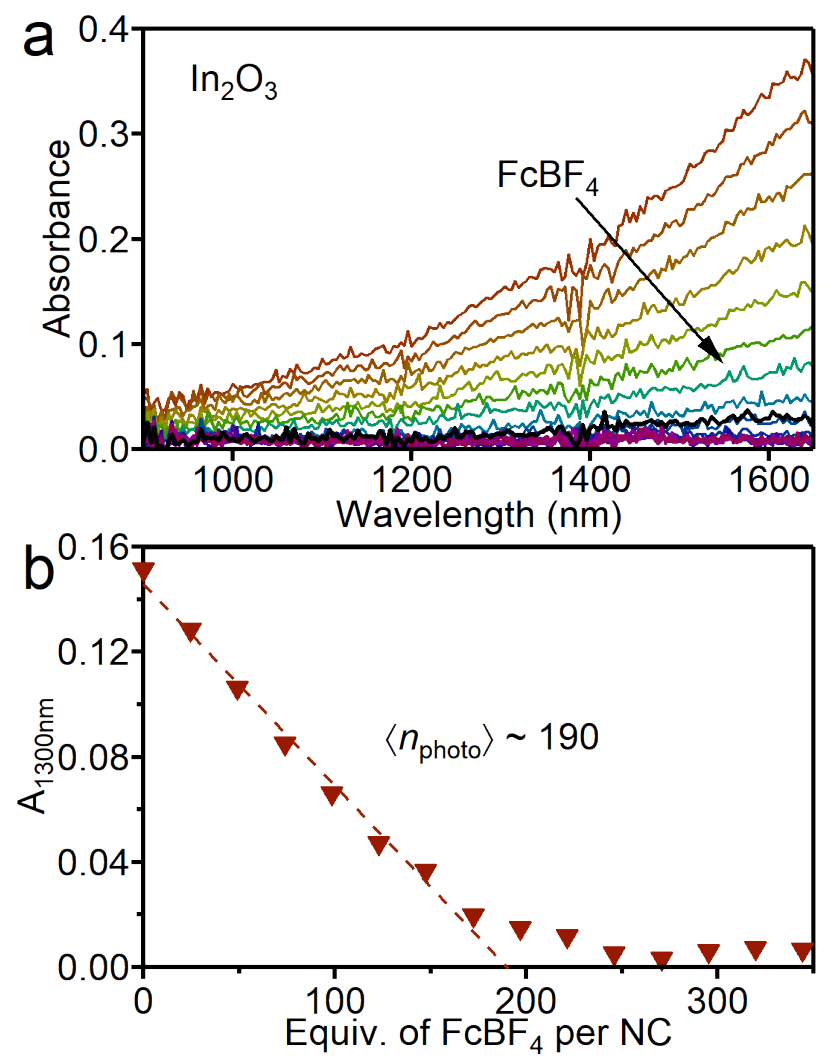

Figure S2. (a) Absorption spectra of a solution of maximally photodoped $d=13.1 \mathrm{~nm} \mathrm{In}_{2} \mathrm{O}_{3}$ $\mathrm{NCs}$, collected at various stages during oxidative titration with $\mathrm{FcBF}_{4}$. The $\mathrm{NCs}_{\text {were suspended }}$ in $0.1 \mathrm{M} \mathrm{TBAPF}_{6} / \mathrm{THF}$. The arrow shows the direction of increasing oxidative equivalents. The black spectrum is the absorption of the as-prepared solution. (b) Absorbance at $1300 \mathrm{~nm}$ plotted against equivalents of $\mathrm{FcBF}_{4}$ added to the maximally photodoped NCs from panel (a). The dashed red line is a fit to the data for values less than 150 . 

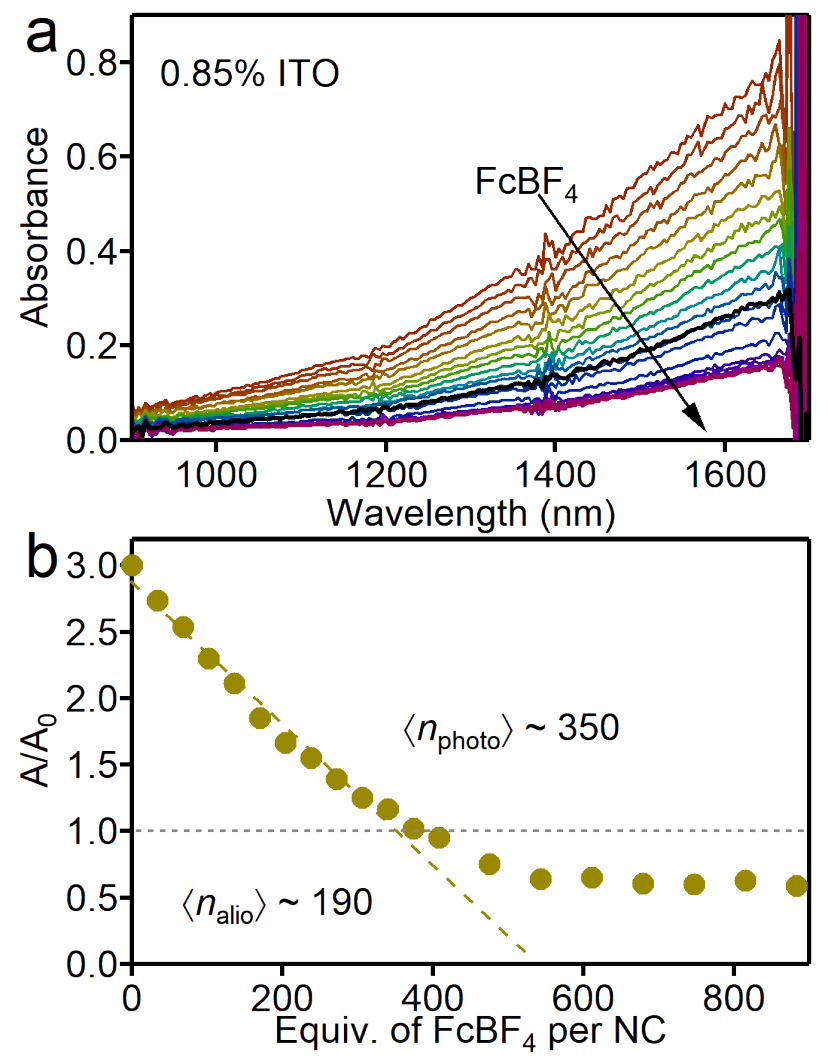

Figure S3. (a) Absorption spectra of a solution of maximally photodoped $d=13.4 \mathrm{~nm}$ ITO NCs containing $0.85 \% \mathrm{Sn}^{4+}$, collected at various stages during oxidative titration with $\mathrm{FcBF}_{4}$. The NCs were suspended in $0.1 \mathrm{M} \mathrm{TBAPF} / \mathrm{THF}$. The arrow shows the direction of increasing oxidative equivalents. The black spectrum is the absorption of the as-prepared solution. (b) The ratio of the absorbance at $1300 \mathrm{~nm}$ to the absorbance of the as-prepared solution at the same wavelength $\left(A / A_{0}\right)$ ), plotted against equivalents of $\mathrm{FcBF}_{4}$ added to the maximally photodoped NCs from panel (a). The gray dotted line at $A / A_{0}=1$ represents the absorption of the as-prepared solution. The dashed gold line is a fit to the data for values of $A / A_{0}$ greater than 1 and is extrapolated to $A / A_{0}=0$. 


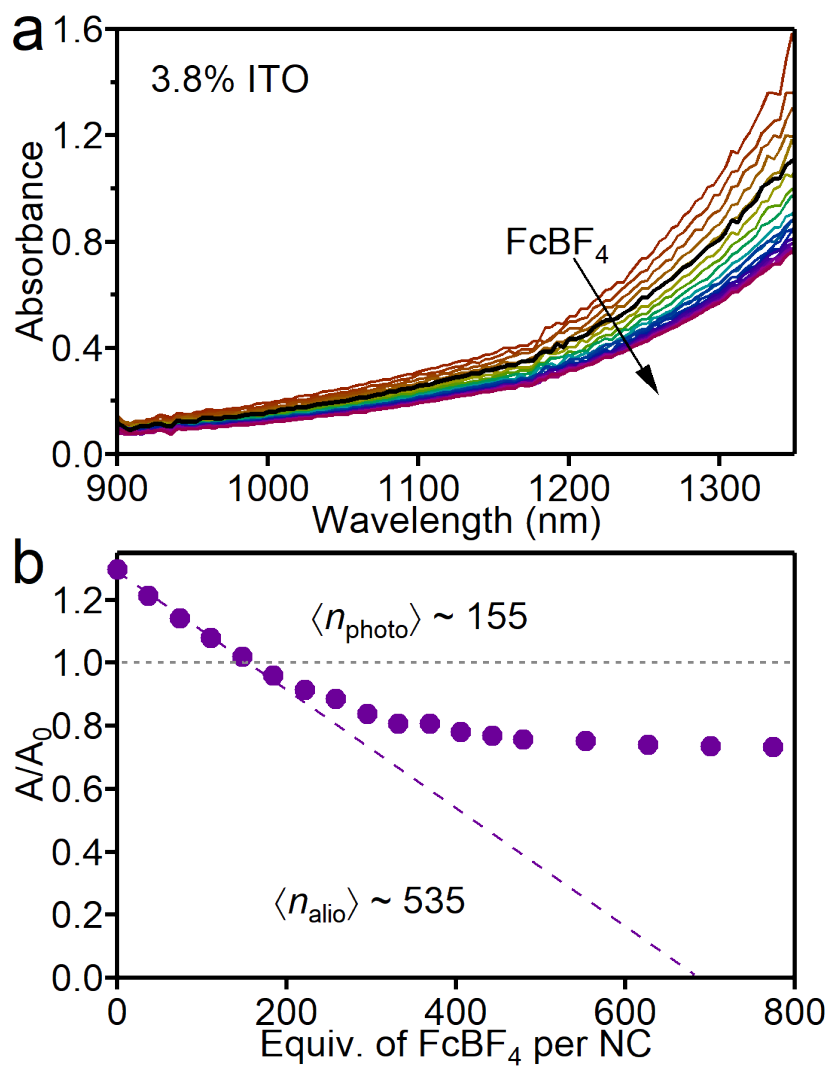

Figure S4. (a) Absorption spectra of a solution of maximally photodoped $d=12.6 \mathrm{~nm}$ ITO NCs containing 3.8\% $\mathrm{Sn}^{4+}$, collected at various stages during oxidative titration with $\mathrm{FcBF}_{4}$. The NCs were suspended in $0.1 \mathrm{M} \mathrm{TBAPF}_{6} / \mathrm{THF}$. The arrow shows the direction of increasing oxidative equivalents. The black spectrum is the absorption of the as-prepared solution. (b) The ratio of the absorbance at $1300 \mathrm{~nm}$ to the absorbance of the as-prepared solution at the same wavelength $\left(A / A_{0}\right)$, plotted against equivalents of $\mathrm{FcBF}_{4}$ added to the maximally photodoped NCs from panel (a). The gray dotted line at $A / A_{0}=1$ represents the absorption of the as-prepared solution. The dashed purple line is a fit to the data for values of $A / A_{0}$ greater than 1 and is extrapolated to $A / A_{0}$ $=0$. 


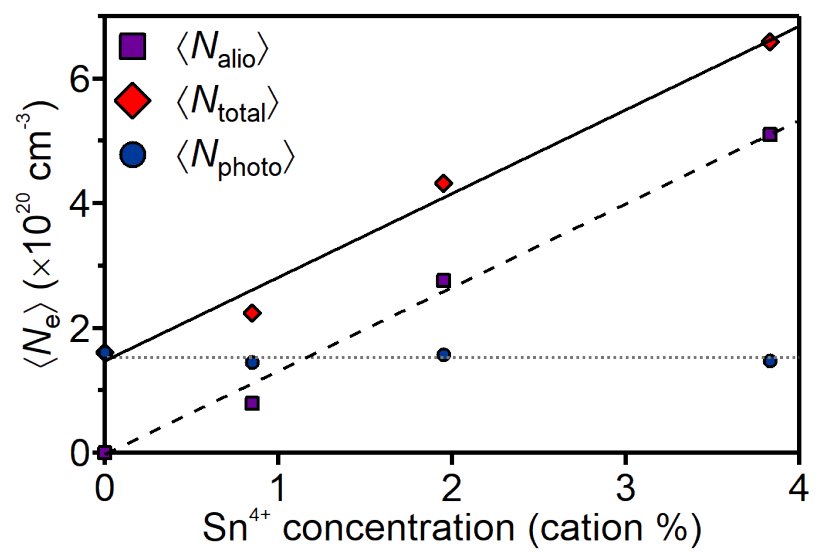

Figure S5. Electron density $\left(\times 10^{20} \mathrm{~cm}^{-3}\right)$ for electrons compensated by aliovalent dopants (purple squares), electrons added by photodoping (blue circles), and total electron density (red diamonds). The dotted gray line at $\sim 1.5 \times 10^{20} \mathrm{~cm}^{-3}$ is a guide to the eye highlighting a constant value of $\left\langle N_{\text {photo }}\right\rangle$ across the range of $\mathrm{Sn}^{4+}$ concentrations. The diagonal dashed line is a guide to the eye showing increasing $\left\langle N_{\text {alio }}\right\rangle$ with increasing $\mathrm{Sn}^{4+}$ concentration, and the solid diagonal line is a guide to the eye showing the total electron density of the maximally photodoped NCs $\left(\left\langle N_{\text {total }}\right\rangle\right.$ $\left.=\left\langle N_{\text {alio }}\right\rangle+\left\langle N_{\text {photo }}\right\rangle\right)$.

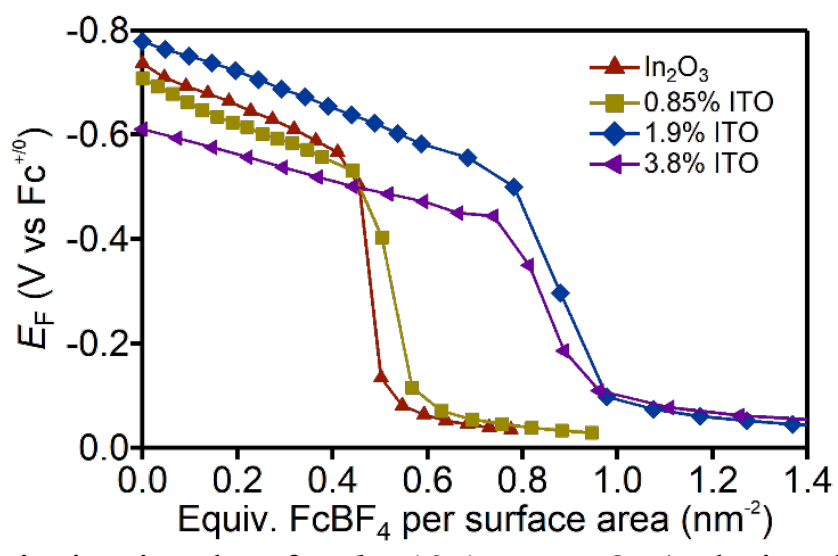

Figure S6. Potentiometric titration data for $d=13.1 \mathrm{~nm} \mathrm{In}_{2} \mathrm{O}_{3}$ (red triangles), $d=13.4 \mathrm{~nm} 0.85 \%$ ITO (gold triangles), $d=14.1 \mathrm{~nm} \mathrm{1.9 \%} \mathrm{ITO} \mathrm{(blue} \mathrm{triangles),} \mathrm{and} d=12.6 \mathrm{~nm} 3.8 \%$ ITO (purple triangles) nanocrystals. The Fermi level $\left(E_{\mathrm{F}}\right.$, in $\left.V v s \mathrm{Fc}^{+/ 0}\right)$ is plotted against equivalents of $\mathrm{FcBF}_{4}$ per unit surface area $\left(\mathrm{nm}^{2}\right)$ added to solutions of maximally photodoped $\mathrm{NCs}$ in $0.1 \mathrm{M}$ $\mathrm{TBAPF}_{6} / \mathrm{THF}$. 


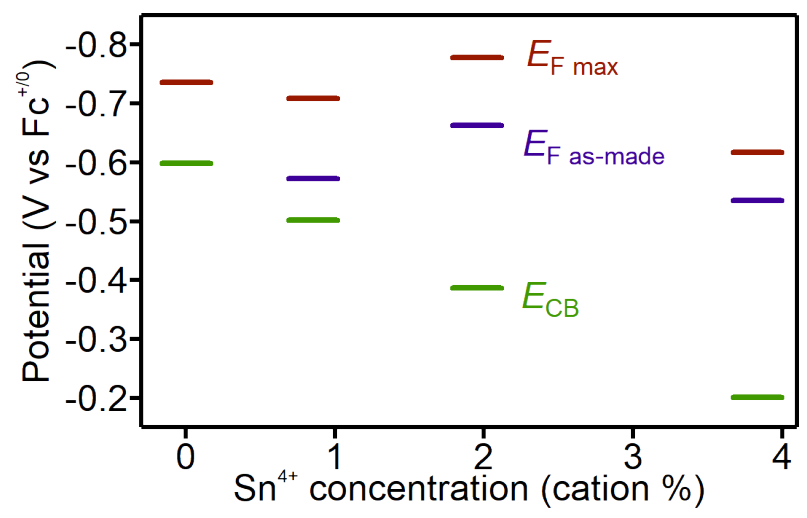

Figure S7. Potentials for $\mathrm{In}_{2} \mathrm{O}_{3}$ NCs with varying $\mathrm{Sn}^{4+}$ content. The red bars indicate the maximum Fermi levels after maximum photodoping. The purple bars indicate the potentials at $A / A_{0}=1$ for the ITO samples. The green bars indicate the CB-edge potentials, derived from extrapolation of the data in Figure 4 to the Fermi level at $A / A_{\max }=0$.

\section{Computational Methods}

Hybrid Quantum-Classical "Charged Sphere" Model. All calculations employ a modified version of the hybrid quantum-classical "Charged Sphere" model described previously. ${ }^{1-2}$ In short, the energetics of $e_{C B}^{-}$within $n$-type spherical NCs are computed quantum mechanically by treating $e_{C B}^{-}$as particles in a finite potential that is classically determined. We first determine the potential $V(r)$ acting on a single-particle wavefunction $\psi(r)$. Using Gauss' law, electron-electron/hole-hole repulsion and electron-cation/hole-anion stabilization are approximated as a mean-field potential. The electron-electron exchange energies are neglected in the model due to their minor contribution to the orbital energies. As a cross-check, we performed an analytical integration of s-p repulsion and s-p exchange on $d=4 \sim 10 \mathrm{~nm}$ spheres using an infinite square potential well (analytical integration is not feasible for a finite well) and found that in all cases the exchange energies are an order of magnitude smaller than repulsions. Therefore, neglecting exchange slightly increases orbital energies but retains the primary qualitative result. 
For a specific configuration of spatially distributed negatively charged particles, we use Gauss' law to calculate $V(r)$ in the Hamiltonian as shown in eq $\mathrm{S} 1$ from the corresponding electric field,

$$
\hat{\mathrm{H}}=-\frac{1}{2} \frac{d^{2}}{d r^{2}}+\frac{l(l+1)}{2 r^{2}}+V(r)
$$

For charged particles, Gauss' law can be written as

$$
\oiint \vec{D} \cdot d \vec{S}=\iiint \rho d V
$$

The left-hand side integrates the electric displacement field over the surface area of an arbitrary Gauss' sphere, and the right-hand side gives the total number of effective charges included in the sphere.

Assuming we only draw Gauss' sphere inside the NC, eq S2 can be expanded as:

$$
\epsilon_{0} \epsilon E(r) 4 \pi r^{2}=\iiint \rho d V
$$

where $\epsilon_{0}$ and $\epsilon$ are the dielectric constants of vacuum and ITO, respectively.

The potential at a distance $r$ is easily determined by integration:

$$
V(r)=\int_{\infty}^{r} E\left(r^{\prime}\right) d r^{\prime}
$$

The same treatment can be applied to derive the potential outside the NC. If the potential $V(r)$ is determined electrostatically, then it should be a continuous function spanning the radial coordinate. In the model, we introduce quantum effects in the form of electron affinity (EA) for the $e_{C B}^{-}$to further confine the particles from escaping the NCs. Therefore, the overall potential $V(r)$ is a piecewise function:

$$
V_{\text {tot }}(r)=\left\{\begin{array}{r}
V_{\text {in }}(r)-E A, r<R \\
V_{\text {out }}(r), r \geq R
\end{array}\right.
$$

Substituting $V_{\text {tot }}(r)$ back into eq $\mathrm{S} 1$ gives the Hamiltonian for calculating the orbital energy (eigenvalue) and wavefunction $\psi(r)$ (eigenvector) of the Fermi electron in the NC through 
simple numerical methods. The electron affinity (EA) and dielectric constants $(\epsilon)$ of the material and of the solvent are required as input parameters in the model calculations. For all ITO calculations, the dielectric constant of the solvent $\left(0.1 \mathrm{M} \mathrm{TBAPF}_{6} / \mathrm{THF}\right)$ is 9.0 . The dielectric constant and EA of ITO are chosen to be the same as for undoped $\operatorname{In}_{2} \mathrm{O}_{3}$, i.e., $\epsilon=12.5$ and EA $=$ $4.1 \mathrm{eV}$.

Figure S8a plots the potentials obtained for uniform $(\rho(r)=$ constant $)$ distributions of active aliovalent dopants at different doping levels in an $R=7 \mathrm{~nm} \mathrm{In}_{2} \mathrm{O}_{3} \mathrm{NC}$, referenced to the result obtained for the undoped $\operatorname{In}_{2} \mathrm{O}_{3}$ NC. The incorporation of aliovalent $\mathrm{Sn}^{4+}$ dopants inside the $\mathrm{In}_{2} \mathrm{O}_{3} \mathrm{NC}$ stabilizes the $\mathrm{CB}$ electron.
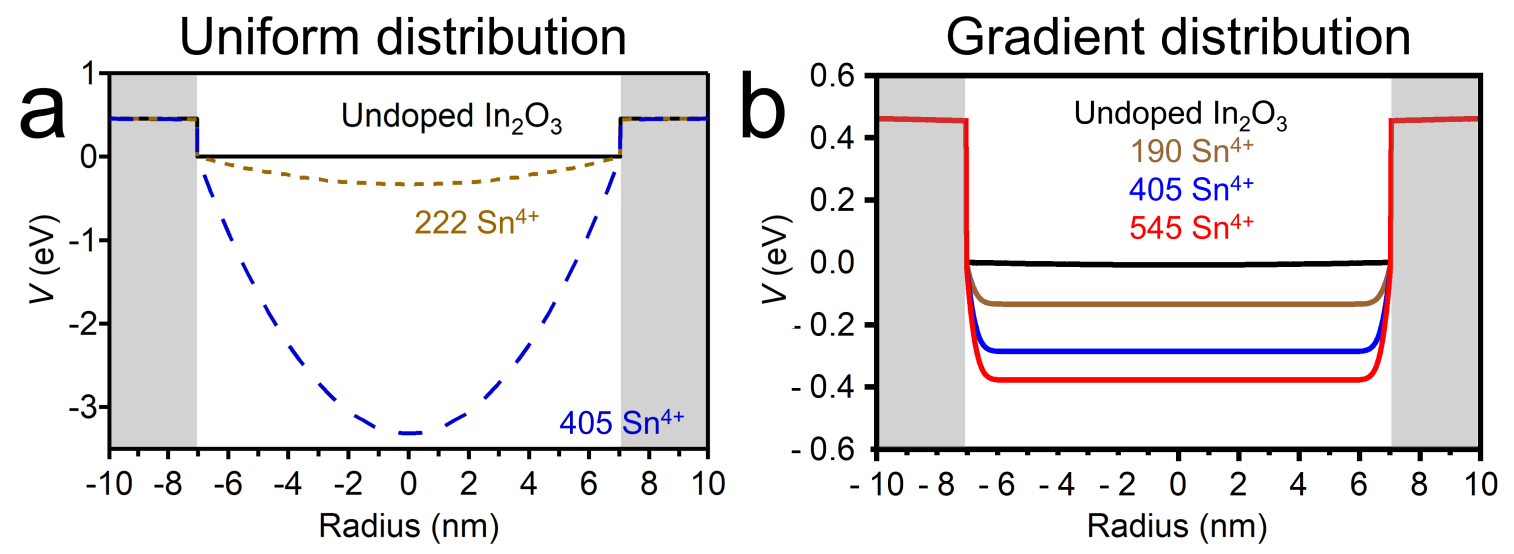

Figure S8. (a) Calculated Fermi-level potential $\left(V_{\mathrm{F}}\right)$ for an $R=7 \mathrm{~nm} \operatorname{In}_{2} \mathrm{O}_{3} \mathrm{NC}$ containing 0 (black), 222 (gold), and 405 (blue) lattice $\mathrm{Sn}^{4+}\left(q^{+}\right) \mathrm{Sn}^{4+}$ dopants distributed uniformly throughout the $\mathrm{NC}$ volume. Equivalent to Fig. 7 of the main text. (b) Calculated potential energies for an $R=7 \mathrm{~nm} \mathrm{In}_{2} \mathrm{O}_{3} \mathrm{NC}$ containing 0 (black), 190 (brown), 405 (blue), and 535 (red) lattice $\mathrm{Sn}^{4+}\left(q^{+}\right)$impurity ions distributed with a Gaussian profile as described by eq S6. In both (a) and (b), $q^{-}=$ 1 to probe the $\mathrm{CB}$-edge potential. The white regions correspond to the $\mathrm{NC}$ interior and the gray regions correspond to the surrounding dielectric. For all calculations, a value of 9.0 is used for the dielectric constant of the solvent $(0.1 \mathrm{M}$ $\mathrm{TBAPF}_{6} / \mathrm{THF}$ ). The dielectric constant and EA of the ITO NCs are chosen to be the same as for $\operatorname{In}_{2} \mathrm{O}_{3}$, i.e., $\epsilon=12.5$ and $\mathrm{EA}=4.1 \mathrm{eV}$. In all calculations, $\mathrm{Sn}^{4+}$ doping is found to stabilize the $\mathrm{CB}$ edge. 
The distribution of lattice $\mathrm{Sn}^{4+}$ inside the $\mathrm{NC}$ also modifies the interior potential. To examine the effects of non-uniform active dopant radial distributions within the NCs, we have modeled various hypothetical distributions for comparison with the uniform distribution of Fig. S8a. For example, one may consider a Gaussian distribution with $\rho(r)$ defined as:

$$
\rho(r)=A e^{-\frac{(r-R)^{2}}{2 \sigma^{2}}}
$$

where $A$ is the normalization factor that preserves the total number of cations and $\sigma$ is the Gaussian width that describes the spread of the dopant distribution away from the NC surface. This distribution represents a growing probability of active dopants at increasing $r$ within the NCs. Figure S8b shows the results of calculations using eq $\mathrm{S} 6$ with $\sigma=0.53 \mathrm{~nm}$, in which most of the active dopants are within two chemical bonds of the NC surface. Shifting aliovalent dopants toward the NC surfaces diminishes the carrier stabilization energies inside the $\mathrm{NC}$ and flattens $V(r)$, ultimately evolving into the potential calculated for surface charge compensation described in the main text.

Dependence of $E_{F}$ on Nanocrystal Radius and Double-Layer Thickness. Eq S7 describes the relationship between the Fermi level and the distance $(\Delta R)$ of charge-balancing cations from the $\mathrm{NC}$ surface. ${ }^{1}$

$$
\Delta E_{F}=\frac{q^{+}}{\epsilon_{0} \epsilon_{\text {out }} R}-\frac{q^{+}}{\epsilon_{0} \epsilon_{\text {out }}(R+\Delta R)}
$$

As noted in the main text, larger values of $R$ will result in a smaller influence of $\Delta R$, resulting in a larger capacitance $\left(C_{r} \propto 1 / \Delta E_{F}\right)$.

Approximation of Surface Dipoles through the Charged Sphere Model. Similarly, the change in Fermi level energy can be described by the surface dipole density $\left(\sigma_{d}\right)$ for a sphere with radius much larger than the surface dipole layer thickness. ${ }^{3}$ 


$$
\Delta E_{F}=\frac{\sigma_{d}}{\epsilon_{0} \epsilon_{\text {out }}}
$$

For values of $R$ much larger than $\Delta R$, it is easily shown that eq $\mathrm{S} 8$ is an approximation of the Charged Sphere model.

$$
\frac{q^{+}}{\epsilon_{0} \epsilon_{\text {out }} R}-\frac{q^{+}}{\epsilon_{0} \epsilon_{\text {out }}(R+\Delta R)}=\frac{q^{+}}{\epsilon_{0} \epsilon_{\text {out }}} \frac{\Delta R}{R(R+\Delta R)} \approx \frac{q^{+} \Delta R}{\epsilon_{0} \epsilon_{\text {out }} R^{2}}=\frac{\sigma_{d}}{\epsilon_{0} \epsilon_{\text {out }}}
$$

\section{References}

(1) Liu, H.; Brozek, C. K.; Sun, S.; Lingerfelt, D. B.; Gamelin, D. R.; Li, X. A Hybrid QuantumClassical Model of Electrostatics in Multiply Charged Quantum Dots. J. Phys. Chem. C 2017, 121, 26086-26095.

(2) Brozek, C. K.; Zhou, D.; Liu, H.; Li, X.; Kittilstved, K. R.; Gamelin, D. R. Soluble Supercapacitors: Large and Reversible Charge Storage in Colloidal Iron-Doped $\mathrm{ZnO}$ Nanocrystals. Nano Lett. 2018, 18, 3297-3302.

(3) Jeong, K. S.; Deng, Z.; Keuleyan, S.; Liu, H.; Guyot-Sionnest, P. Air-Stable n-Doped Colloidal HgS Quantum Dots. J. Phys. Chem. Lett. 2014, 5, 1139-1143. 